\title{
De Franz Boas a Ángel Palerm
}

\author{
Marisol Pérez Lizaur
}

Se muestra cómo la metodología para enseñar investigación de trabajo de campo, desarrollada por Franz Boas, pasó de una generación de estudiantes a otra y cómo cada una enriqueció el modelo hasta que Palerm lo transformó en una metodología específica de formación de estudiantes de antropología en el campo en México. Se presenta dicha genealogía y las redes académicas a su alrededor. Boas trabajó sólo con un ayudante. En la Universidad de Columbia enseñó a Kroeber y a Lowie, quienes repitieron el modelo de enseñanza en Berkeley con Kelly, sin acompañarla al campo. Kelly realizó trabajo de campo en México y formó a estudiantes en la Escuela Nacional de Antropología e Historia. Palerm aprendió de ella y luego instrumentó su metodología de enseñanza en la Universidad lberoamericana en México.

PALABRAS CLAVE: trabajo de campo, metodología de enseñanza de trabajo de campo, Ángel Palerm, Isabel Kelly, Universidad lberoamericana, metodología de trabajo de campo

\section{From Franz Boas to Ángel Palerm}

We show how the methodology for teaching fieldwork research, developed by Franz Boas, passed from one generation of students to the next and how each generation enriched the model, until Palerm transformed it into a specific methodology for the training of anthropology students in the field in Mexico. We present this academic genealogy, and the academic networks around it. Boas worked with just one assistant. At Columbia University he taught Kroeber and Lowie, who reproduced Boas' teaching model at Berkeley and trained Kelly without accompanying her to the field. Kelly did fieldwork research in Mexico and trained students from the National School of Anthropology and History. Palerm learned from her and subsequently established his teaching methodology at the Universidad Iberoamericana in Mexico.

KEYWORDS: fieldwork, teaching fieldwork methodology, Ángel Palerm, Isabel Kelly, Universidad Iberoamericana, fieldwork methodology

Marisol Pérez Lizaur: Universidad Iberoamericana, Distrito Federal, México marisol_perez@prodigy.net.mx 


\section{INTRODUCCIÓN}

$\mathrm{E}$ n este trabajo mostraré los antecedentes de la metodología de enseñanza de la investigación de campo instrumentada por Ángel Palerm en la Universidad Iberoamericana (UIA), en la ciudad de México. Indagué en documentos bibliográficos y de archivo sobre la metodología y las relaciones maestro-alumno en el campo, desde Franz Boas hasta Palerm. Para ilustrar este proceso partí de mi experiencia como alumna del propio Palerm, entrevisté a algunos de mis compañeros y maestros, y busqué en libros - sobre todo en Kelly, Harris, Stocking y Steward- descripciones precisas sobre la manera en que se formaban los estudiantes en el campo. Sin embargo, para mi gran sorpresa, salvo Evon Vogt (1994), director del Chiapas Project de la Universidad de Harvard, formado en la Universidad de Chicago, ninguno es preciso acerca del tema y en general lo dan por entendido. Mi interés se centraba en cómo Kelly había aprendido de
Kroeber y cómo había transmitido este aprendizaje a sus estudiantes. Dada la falta de información recurrí a Robert Kemper, curador del archivo de Isabel Kelly, quien generosamente me facilitó cerca de mil cartas. En ellas no sólo encontré un recuento de su metodología de investigación, sino un relato detallado de la formación de los estudiantes en el campo.

El análisis de los materiales bibliográficos y de archivo reveló que la metodología de la enseñanza de la investigación de campo ha variado desde la transmisión de Boas a sus alumnos y de ellos en adelante. La comparación pone al descubierto que cada investigador desarrolla el trabajo de campo de acuerdo con su intuición, conocimiento y experiencia y que cada uno aporta algo nuevo o distinto a la manera en que fue formado. Cada investigador en su calidad de maestro transmite su conocimiento y su experiencia personal con base en la que tuvo un día como alumno. Esta transmisión del conocimiento no es lineal, en ella influyen la creatividad y la práctica de maestros y alumnos, así como las relaciones entre ambos, condicionadas por el entorno institucional en las cuales ocurren. Este proceso está determinado en gran medida por la personalidad e intereses, conocimientos académicos, inquietudes teóricas y políticas, experiencias humanas y de trabajo de los participantes, al igual que por el contexto histórico, político e institucional que lo enmarca. Así, la metodología de la enseñanza de la investigación de campo varía entre maestros, pero al mismo tiempo aparece un cúmulo de conocimientos y experiencias que se transmite de generación en generación.

En el esquema que adjunto presento una especie de genealogía académica de Ángel Palerm. Se muestran las conexiones maestro-alumno, las influencias o relaciones entre académicos, instituciones o movimientos sociales; las orientaciones teóricas de los investigadores; algunas de las instituciones en las que trabajaron; las metodologías o formas en que desarrollaban o enseñaban el trabajo de campo y las influencias estadounidenses, europeas y mexicanas que confluyeron en Palerm, quien concretó su metodología de enseñanza en la UIA. Es probable que no sea un reflejo perfecto de lo que ocurrió en realidad, pero sirve como guía para entender lo que a continuación escribo.

\section{FRANZ BOAS}

Comienzo esta crónica con Franz Boas, reconocido en la disciplina como el "padre de la metodología de la investigación de campo". En primer lugar hay que recordar que Boas fue un gran promotor de la institucionalización de la enseñanza de la antropología, pero al inicio de su carrera como investigador en el campo trabajó solo. Por otro lado, su formación y orientación humanista y en las ciencias naturales caracterizó su trabajo como antropólogo, así como su forma de realizar la investigación de campo (Stocking, 1983). Sus cartas, publicadas por Stocking (1983), dejan ver a un investigador de campo solitario, que trabajó con un asistente nativo y que registraba sus observaciones y entrevistas en largos y 


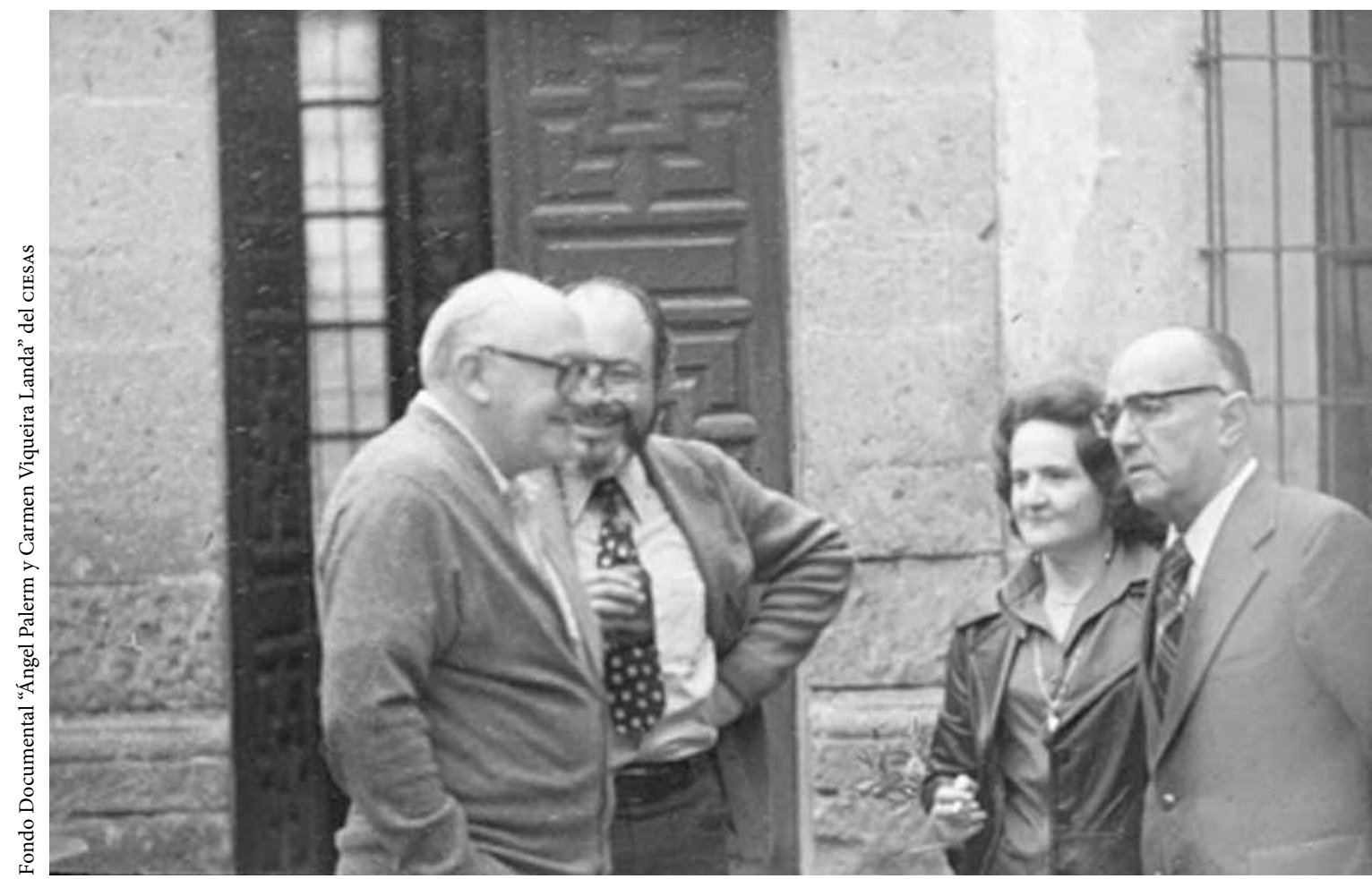

Ángel Palerm, Guillermo Bonfil, Susana Glantz y Gonzalo Aguirre Beltrán en Casa Chata, ca. 1973.

detallados diarios de campo y cartas. Según Lowie, el más fiel de sus alumnos (Harris, 1978: 248), nunca fue capaz de integrar sus trabajos de manera ordenada.

Su personalidad y su orientación teórica y metodológica lo llevaron a realizar etnografías muy precisas, en las que verificaba los datos con sumo cuidado y respetaba el particularismo histórico. Con dicho bagaje formó a sus estudiantes en la Universidad de Columbia de 1896 hasta 1941, entre muchos otros: Robert Lowie, Alfred Kroeber, Melville Herskovits, Ruth Benedict, Margaret Mead, Fay-Cooper Cole, Edward Sapir y el mexicano Manuel Gamio, "el padre de la antropología mexicana”, con quien fundó en 1910 en la ciudad de México la Escuela Internacional de Arqueología y Etnología. Posteriormente, Kroeber y Lowie enseñaron en la Universidad de California en Berkeley; Cole y Sapir en Chicago, Herskovits en North Western y Gamio en la Escuela Nacional de Antropología e Historia
(ENAH) en México (Stocking, 1983; Harris, 1978: 219; Rutsch, 2007: 250).

\section{KROEBER}

Según Harris, la formación original de Kroeber en literatura inglesa, combinada con las enseñanzas de su maestro Boas, marcaron su trabajo teórico y metodológico. De Boas aprendió la importancia del trabajo de campo prolongado y detallado, así como la búsqueda de la precisión en los datos y de la objetividad (Stocking, 1996; Harris, 1978). Como maestro en Berkeley, su interés principal fue enseñar a sus alumnos a realizar etnografías minuciosas con la finalidad de rescatar las culturas de los pueblos de California (Fowler y Kemper, 2008: XI). Sin embargo, a decir de Harris, a diferencia de su maestro, para quien cada cultura tenía que entenderse y 
explicarse en sus propios términos sin considerar sus antecedentes históricos, Kroeber aspiraba a entender y explicar las grandes tendencias históricas o majestic forces. Con este afán perdió el interés de verificar la información (Harris, 1978: 288). Es decir, Kroeber buscaba encontrar a través de la investigación de campo la presencia de patrones constantes que, según él, aparecían en el desarrollo cultural. En palabras de Stocking:

El principal interés de Kroeber era encontrar "las grandes fuerzas autónomas" que explicaban el desarrollo cultural". Para él dichas fuerzas solamente podían ser aprehendidas a través de los elementos fenomenológicos producidos por ellas. Dicho de otra manera, Kroeber, de acuerdo con su propio criterio, "trabajaba con datos concretos" (Stocking, 1996: 268).

Su devoción por los particulares estaba al servicio del "reconocimiento de los patrones" (Stocking 1996: 268). ${ }^{1}$

En Berkeley formó a sus estudiantes de acuerdo con estos principios y los mandaba al campo solos para elaborar etnografías pormenorizadas con el afán de detectar patrones y realizar comparaciones. A pesar de este interés por las etnografías detalladas, según su alumna Isabel Kelly, enviaba a los estudiantes al campo sin mayor preparación y sin decirles qué hacer ni cómo (carta a Foster, 1950). ${ }^{2}$ Kroeber enseñaba en el Departamento de Antropología de la

\footnotetext{
1 "Kroeber's ultimate concern was with 'majestic forces', these forces could only be apprehended through the phenomenal elements that they produced. Thus Kroeber was, by his own estimation 'a worker in concrete data"' (Stocking, 1996: 268).

"His devotion to particulars was in the service of 'pattern recognition"' (Stocking, 1996: 268) [traducción libre de la autora].

${ }^{2}$ En una entrevista, Hugo G. Nutini —estudiante de antropología en la Universidad de California en Los Ángeles en la década de 1950 - señaló que su maestro Pedro Carrasco — quien estudió en la Universidad de Columbia y en la ENAH (Nutini, Carrasco y Taggart, 1976) - lo mandó al campo solo, en Tlaxcala, sin decirle qué hacer. Lo primero que se le ocurrió fue levantar un censo. El resultado de su investigación fue su tesis doctoral, publicada como la etnografía de San Bernardino Contla. Posteriormente, Nutini impartió clase en la UiA y llevó a David Robichaux al campo. Actualmente Robichaux es el maestro responsable de las prácticas de campo dirigidas de los estudiantes de esa universidad.
}

Universidad de Berkeley, con Lowie. Este último, reconocido como el académico más cercano a la tradición boasiana, se dedicó a realizar la etnografía de los pueblos de las Grandes Planicies, especialmente los cuervo. Tenía un particular interés por la cultura material, aunque era un especialista en la organización social y la religión, y fue un comparativista consumado (Fowler y Kemper, 2008: XI). Otros maestros eran Gifford, un autodidacta formado en el museo universitario con intereses arqueológicos, y Carl A. Sauer, geógrafo, cuya especialidad era la geografía cultural y la historia. Según Fowler y Kemper, los estudiantes recurrían a Gifford y Sauer, que los llevaban a hacer recorridos de campo durante los cuales probablemente les enseñaron algunas técnicas. Por el contrario, Kroeber mandaba a sus estudiantes desde la licenciatura a llevar a cabo investigación antropológica sin decirles cómo desarrollarla. Al parecer, no era muy cercano a ellos, sobre todo si eran mujeres (Fowler y Kemper, 2008: XIII).

\section{ISABEL KELLY}

Isabel Kelly estudió en Berkeley en este entorno académico en el que se enfatizaba la importancia de los estudios etnográficos y la comparación, es decir, la etnología. ${ }^{3}$ Sus compañeros más cercanos fueron Cora Dubois, Julian Steward, Ralph Beals y Lloyd Warner. Como estudiante se esforzó por obtener formación de campo en arqueología y realizó trabajo de campo etnográfico entre los paiutes del norte. A partir de entonces, hizo trabajo arqueológico y de campo con una gran preocupación por la historia y la ecología con diversos grupos estadounidenses y del noroeste mexicano, apoyada por la National Science Foundation y diversas universidades y organizaciones norteamericanas (Fowler y Kemper,

\footnotetext{
3 Ángel Palerm, en su Introducción a la teoría etnológica (1967), critica el particularismo histórico de Boas y las interpretaciones del súper orgánico de Kroeber. Sin embargo, hace hincapié en que el objetivo de la antropología como ciencia es realizar comparaciones para crear una teoría etnológica.
} 
2008). Para ella, era primordial integrar los datos etnográficos de forma coherente, para encontrar explicaciones teóricas. Es decir, su objetivo era elaborar etnografías muy detalladas que sirvieran de base para el análisis y la teoría (Fowler y Kemper, 2008: XVIII).

En 1939, con el auxilio de Sauer, consiguió respaldo para estudiar la arqueología del noroeste de México. Desde entonces vivió en México, donde llevó a cabo investigación arqueológica principalmente en Sonora, Sinaloa y Michoacán, así como estudios etnográficos entre los totonacos y de antropología aplicada con los nahuas y otros grupos. Su labor es testimonio de su gran interés por la arqueología y por la cultura material. En 1946 George Foster la recomendó a Julian Steward para el puesto de antropóloga responsable del Smithsonian Institute of Social Anthropology en la ciudad de México, con el apoyo del cual desarrolló gran parte de su trabajo. Entre sus obligaciones como antropóloga responsable, impartía clases en la licenciatura en antropología de la ENAH, donde formó estudiantes en el campo (Fowler y Kemper, 2008).

Su primer proyecto de investigación para el instituto en colaboración con la ENAH fue el estudio de los totonacos de Tajín, en Veracruz. Su primera salida a campo con estudiantes fue durante el primer semestre de 1947, con financiamiento de ambas instituciones (Fowler y Kemper, 2008). Su objetivo en estas salidas era enseñar a los estudiantes a realizar investigación de campo de la manera en que "¡ella se quejaba de no haber recibido nunca de Kroeber!" (Fowler y Kemper, 2008: XXXIV). En otras palabras, ella diseñó la metodología de investigación de campo y de su enseñanza con base en sus conocimientos y experiencia, adecuada al medio institucional de la ENAH. ${ }^{4}$

La lectura de las cartas de Kelly a Foster, director del Smithsonian Institute of Social Anthropology, de

\footnotetext{
${ }^{4}$ Recordemos que en la ENAH existía una tradición de investigación de campo con raíces en los trabajos realizados por los primeros investigadores en antropología con formación en las ciencias naturales, como Nicolás León, y reforzada por Boas y sus discípulos, incluyendo a Gamio (Rutsch, 2007).
}

1946 a 1952, revela cómo organizaba la actividad de la enseñanza de la investigación etnográfica. En primer lugar, dirigía un seminario en la ENAH sobre los totonacos de Tajín, en el que participaban estudiantes de la licenciatura interesados en su proyecto. Discutían la bibliografía pertinente, los materiales de campo y los reportes, así como los artículos derivados de la investigación. El seminario constituía el espacio donde se reclutaba a los estudiantes para la investigación de campo, quienes iban becados por la ENAH y por el Smithsonian Institute.

Los primeros semestres de 1947, 1948 y 1949 Kelly realizó trabajo de campo entre los totonacos de Tajín. En las tres ocasiones llevó consigo a tres estudiantes, uno de ellos fue Ángel Palerm, quien se incorporó al proyecto en enero de 1948. Financiada por el Smithsonian, montó una casa de campo en Papantla para albergarse con los estudiantes. Se ocupó personalmente de equipar la casa, de comprar y llevar comida y de supervisar el bienestar de todos con el apoyo de Chema, su cocinero personal. Adaptada al medio mexicano, en el que los padres tenían sumo cuidado con sus hijas, en una de las cartas relata que una de sus tareas importantes era ¡hacerla de chaperón de las estudiantes! ${ }^{5}$

Como primera tarea, los estudiantes tenían que hacer un mapa de la localidad y levantar un censo. Por experiencia, más adelante menciona que era mejor ir de casa en casa para recabar la información de las familias y establecer relaciones con los lugareños. Para Kelly estas relaciones eran primordiales en el campo, pues permitían conseguir informantes clave y realizar entrevistas. Ella concedía mayor importancia a la información obtenida por esta vía que a la observación. ${ }^{6}$ Sin embargo, advierte que enfrentaron el problema de que ninguno de los investigadores y estudiantes hablaba totonaco y tuvieron que emplear traductores.

\footnotetext{
5 "Mis padres nunca me dejaron ir a prácticas de campo, hasta que Ángel Palerm y Carmen Viqueira fueron a hablar con ellos" (comentario de la autora).

${ }^{6}$ Boas concedía una gran importancia a las entrevistas con los informantes clave, según sus cartas, publicadas por Stocking.
} 
Parte fundamental de la investigación era el registro en fotos y películas, así como la recolección y la clasificación de plantas. Las cartas muestran su gran interés por ellas, en especial las domésticas. En 1949 invitó a Sauer a visitarlos a Papantla, donde enseñó a los estudiantes a investigarlas y clasificarlas. Otra tarea relevante era escribir las notas de campo en un diario y ordenar fichas con base en la Guía de Murdock. ${ }^{7}$ Ella colaboraba en la recopilación de la información y diario revisaba las notas de campo y las fichas, palabra por palabra. Era terriblemente exigente con la precisión de los datos y su verificación. Además de este trabajo en grupo, los estudiantes escogían un tema personal de investigación sobre el que debían presentar un reporte. Por ejemplo, de acuerdo con su formación e intereses, Palerm buscó y revisó los archivos locales, y aparte de ese trabajo de carácter histórico escribió un capítulo sobre la "Danza de los Negritos" para ser incluido en el libro sobre Tajín que, refiere Kelly, envió a Herskovitz para su revisión.

Para Kelly era importante enseñar a los estudiantes a analizar la información y a redactar reportes y artículos académicos, lo cual incluía la elaboración de cuadros muy complejos. Sus cartas dejan ver que los estudiantes tenían claro que su trabajo de investigación era para el proyecto y que la información generada estaría disponible para los investigadores del Instituto Nacional de Antropología e Historia (INAH). Al regresar de Tajín, Kelly y sus estudiantes se reunían en el seminario semanal en la ENAH y discutían los materiales y los reportes. Aunque en teoría estos seminarios tenían una duración de dos horas, en la mayoría de las ocasiones se extendían hasta cuatro. De manera adicional, Kelly corregía y trabajaba sobre los reportes y los capítulos escritos por los estudiantes. En sus cartas aparece una queja continua sobre la falta de conocimientos del español por parte de los estudiantes.

\footnotetext{
${ }^{7}$ La primera edición se publicó en 1937; la segunda, en 1942 (Palerm y Palerm, 1976).
}

\section{ÁNGEL PALERM ${ }^{8}$}

Palerm inició sus estudios en Ibiza con un tutor de manera básicamente informal, ${ }^{9}$ es decir, fue casi autodidacta y un ávido lector. Interrumpió sus estudios en 1936 para incorporarse como voluntario al ejército republicano español, donde llegó a desempeñarse como jefe de Estado Mayor de una de las brigadas internacionales, en las que tuvo contacto cercano con los anarcosindicalistas (Alonso, 2008). Cuando los republicanos perdieron la guerra, salió como exiliado a México, a donde arribó a mediados de 1939 “y se dedicó a la actividad política que le llevó a replantearse problemas básicos sobre la línea a seguir en las transformaciones revolucionarias" (Viqueira, 2000: 120). Su experiencia y su avidez por la teoría "lo llevaron a romper con toda filiación política" y a hacerse preguntas sobre la historia y los movimientos sociales (Viqueira, 2000: 120).

En 1946 logró ingresar a la carrera de historia en la Facultad de Filosofía y Letras de la Universidad Nacional Autónoma de México (UNAM), donde conoció a don Pablo Martínez del Río. Su intención era "completar la carrera de historia con las optativas de antropología, y terminar antropología con las optativas de historia" (Viqueira, 2000: 120). En enero de 1948, por recomendación de Martínez del Río, quien impartía clases tanto en la ENAH como en la UNAM, Isabel Kelly lo reclutó para integrarse al equipo de estudiantes del Proyecto Tajín. Según Viqueira (2000: 121), "antes de partir al campo, Ángel leyó apresuradamente la Antropología general, de Kroeber, y la Historia de la etnología, de Lowie”.

\footnotetext{
${ }^{8}$ Agradezco profundamente al arquitecto Arturo Pérez Rivera por la elaboración del esquema sobre la genealogía académica de Ángel Palerm.

${ }^{9}$ Según una entrevista con Alba González Jácome y el escrito de Carmen Viqueira (2000), su educación informal lo llevó a dudar de la educación formal e institucionalizada. Esta característica explica en gran medida su búsqueda de flexibilidad en la enseñanza, a pesar de que fue un creador, al igual que Boas, de instituciones de formación de antropólogos.
} 


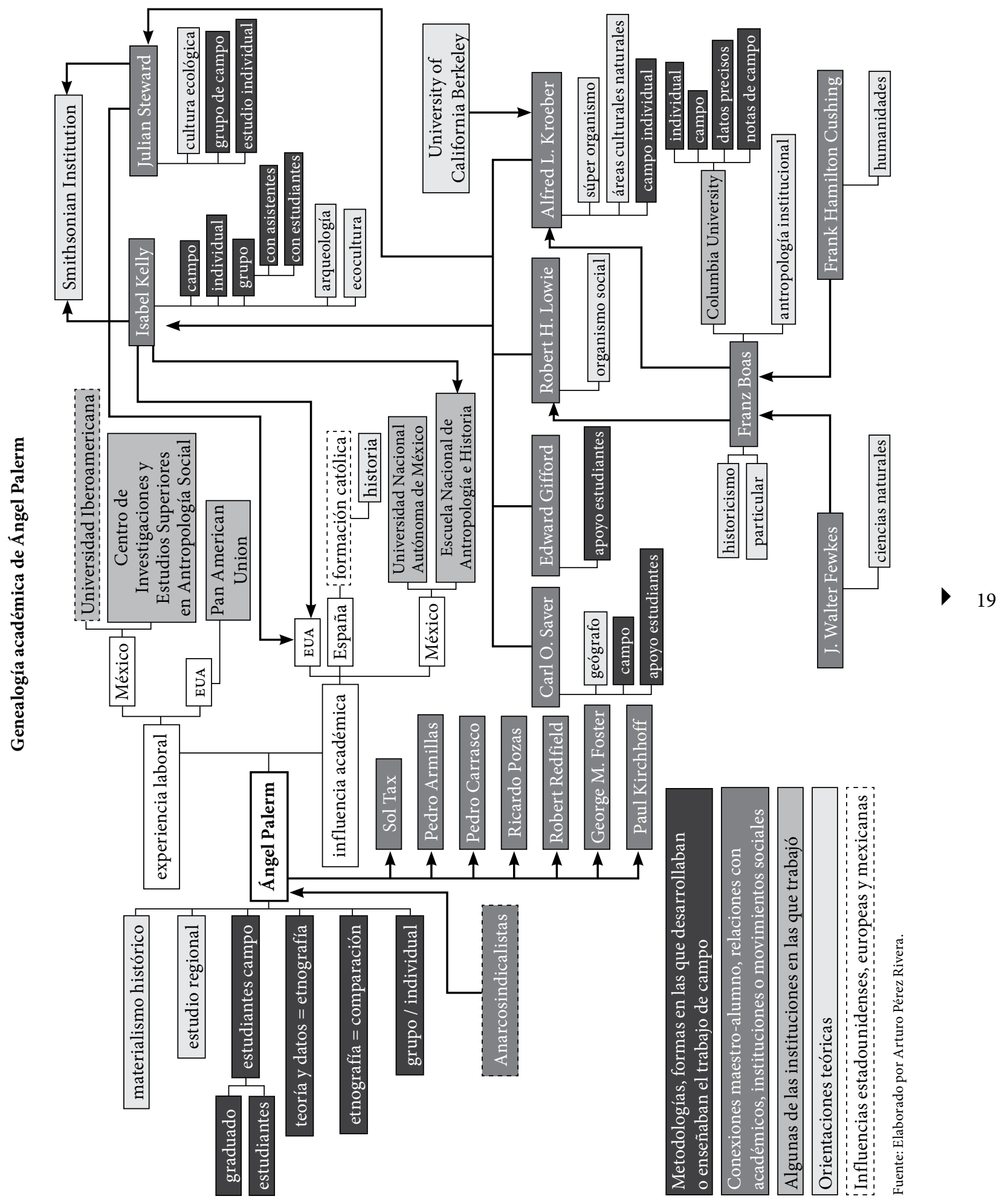


En las cartas de Kelly a Foster de enero de 1948, ella le informa haber contratado a Palerm para el Proyecto Tajín y dice estar sorprendida por su preparación, su forma de pensar y de escribir, muy distinta del común de los estudiantes mexicanos. En varias de las cartas comenta que al principio Palerm le causó la impresión de no ser apto para el campo, pero que al poco tiempo se movía con facilidad y establecía buenas relaciones, sabía tratar y obtener información de los informantes, asistía a las bodas y fiestas y jugaba basquetbol. También reconoce su habilidad para hacer mapas. ${ }^{10}$ Pero lo que más llama la atención de Kelly es la capacidad de Palerm de supervisar el trabajo de los demás estudiantes y de plantear ideas, líneas y temas de investigación. Desde el inicio sugirió realizar una prueba psicológica a las mujeres (Kelly a Foster, enero de 1948). ${ }^{11}$ En una carta a Foster de abril de 1948, Kelly detalla que lo había nombrado asistente con un salario pagado por la Smithsonian Institution, a pesar de que la fundación no cubría ese gasto de manera regular.

Palerm volvió a Tajín en 1949 y en una de las cartas a Foster Kelly precisa: "Cuanto más veo de Palerm, mejor pienso de él como investigador... Con certeza, él es la blanca esperanza de la etnología mexicana" (Kelly a Foster, 3 de enero de 1949). ${ }^{12}$ Varios estudiantes escribieron capítulos para el libro sobre los totonacos, pero Kelly menciona claramente que quería que Palerm fuera coautor del mismo. Y así fue. El proceso de escribir The Tajin Totonac, publicado por la Smithsonian Institution en 1952, no fue tan sencillo. En enero de 1951 Kelly se queja con Foster acerca de algunos errores de Palerm en unas tablas, lo que retrasó el reporte final más tiempo del debido. A raíz de ello, Kelly decide que el segundo volumen no será en coautoría con él. Sin embargo, lo llevará de vuelta al campo en un nuevo

\footnotetext{
${ }^{10}$ En el campo nos mencionó que aprendió a leer y hacer mapas durante la guerra.

${ }^{11}$ Aparentemente la idea de realizar esta prueba fue sugerencia de Carmen Viqueira, su esposa, quien entonces estudiaba psicología.

12 "The more I see of Palerm, the better I think of him as an investigator... He certainly is the white hope of Mexican ethnology".
}

proyecto sobre la salud y la nutrición entre los nahuas de la Sierra de Puebla, también financiado por la Smithsonian Institution. Al llegar al campo, lo primero que hará Palerm será ir a la iglesia a solicitar ver los archivos, igual que en Tajín. Kelly da mucha importancia a la historia y a la relación del hombre con la naturaleza.

Las cartas de 1951 relatan que se habían presentado diferencias graves entre Kelly y Palerm. Desde el problema de los cuadros, ella pierde la confianza en su asistente y él se aparta de su maestra. Las desavenencias entre ambos impiden que se publique el segundo tomo del libro sobre los totonacos de Tajín. ${ }^{13}$ Esto no evitó que Palerm, al final de su vida en 1979, dijera a Marisol Alonso: "aprendí el rigor del trabajo de campo, la metodología y la reflexión colectiva. Los otros profesores me habían dado muchas cosas, pero lo que suele llamarse el oficio lo aprendí con Isabel" (García, 2000: 16).

En 1952 Palerm fue a Washington como editor del Boletín de Ciencias Sociales de la Unión Panamericana recomendado por el antropólogo físico de origen español Juan Comas. Este trabajo le permitió leer y conocer la literatura antropológica más reciente y establecer relaciones con muchos colegas. Conoció a Steward, Wittfogel y Beals, y se hizo amigo de los alumnos de Julian Steward, especialmente de Eric Wolf, Sidney Mintz, John Murra y William Sanders. Construyó una amistad especial con Wolf (1972), con quien realizó una investigación sobre la región

\footnotetext{
${ }^{13}$ Visto a la distancia, las desavenencias entre la maestra y su alumno pueden tener muchas interpretaciones. No recuerdo que Palerm ni Viqueira aludieran al hecho. Sin embargo, la lectura de las cartas de Kelly, y mi interpretación personal como estudiante de Palerm y Viqueira, me sugieren que Ángel Palerm nunca aceptó ser coautor de un libro elaborado con materiales de campo obtenidos en gran medida por él y sus compañeros. Tanto Palerm como Viqueira defendían que en un proyecto en el que trabajaban estudiantes y maestros juntos todos fueran autores y que el profesor figurara como "investigador principal" (Viqueira, 2000). Otra interpretación posible, ligada a lo anterior, es que Palerm haya querido interpretar y analizar la información obtenida con una visión distinta a la de Kelly. Recordemos que Ángel Palerm buscaba respuestas históricas, mientras Kelly las buscaba más cercanas al particularismo histórico de Boas.
} 
de Texcoco en México (García, 2000; Viqueira, 2000; Alonso, 2008).

En 1954 participó en una reunión organizada por la Organización de las Naciones Unidas para la Educación, la Ciencia y la Cultura (unesco) y la Organización de Estados Americanos (oEA) en Costa Rica con el fin de elaborar un plan para apoyar con recursos internacionales la expansión y el mejoramiento de la enseñanza de las ciencias sociales en la región latinoamericana, como una herramienta fundamental para el desarrollo. Allí, con Gonzalo Aguirre Beltrán, propuso un proyecto para desarrollar la investigación y la enseñanza de la antropología en México. El objetivo de dicho plan era formar investigadores investigando, por lo que el trabajo de campo era esencial (Viqueira, 2000: 115-116).

De 1961 a 1965 Palerm fue director del Departamento de Asuntos Sociales de la Unión Panamericana. Durante su estancia en Washington obtuvo el doctorado en planificación. Regresó a México en 1965 con la intención de implementar en la ENAH un proyecto de formación de antropólogos sociales basado en el que había desarrollado con Aguirre Beltrán a partir de la Mesa Redonda de Costa Rica. Parte importante de este proyecto fue también su experiencia como estudiante, investigador y administrador (Viqueira, 2000: 120). La rigidez que imperaba en la ENAH impidió que el proyecto se concretara y Palerm presentó e instrumentó su proyecto en la UIA (Viqueira, 2000: 121122; Alonso, 2008). ${ }^{14}$

Según Viqueira, "la supervisión minuciosa y formativa, y la discusión del proyecto entre el investigador principal y los estudiantes, fue una de las cosas que, convencido, lo formó como antropólogo" (Viqueira, 2000: 121). Su propuesta de llevar a los estudiantes al campo desde el inicio, como sucedía en la ENAH desde la década de 1940, cuando él fue estudiante, tenía dos propósitos: "uno, aprender el método antropológico llevando a cabo investigación, y otro, permitir un diagnóstico temprano de aquellos estudiantes que

${ }^{14}$ Universidad de los jesuitas en la ciudad de México. realmente permanecerían en la antropología [...] Ángel Palerm hacía notar que aquel al que no le guste el trabajo de campo, no puede ser antropólogo".

\section{LA PRIMERA PRÁCTICA DE CAMPO DE LOS ESTUDIANTES DE ANTROPOLOGÍA DE LA UNIVERSIDAD IBEROAMERICANA (1965) ${ }^{15}$}

Invitado por los doctores Felipe Pardinas y Luis González, ambos antropólogos y jesuitas, Palerm impartió en 1965 un curso de planificación para los estudiantes de tercer año de la licenciatura en antropología de la UIA. De acuerdo con Luis González, director de la carrera, decidió llevarnos al campo para poner en práctica su proyecto de enseñanza de la antropología, es decir, de formar investigadores investigando. Optó por llevarnos a la región de Texcoco, cercana a la ciudad de México. Su objetivo era no sólo enseñarnos a investigar, sino también continuar con el proyecto que había realizado en colaboración con Eric Wolf en torno al Acolhuacan septentrional, sobre la ecología y el papel de la distribución del agua en la integración de la región (Palerm y Wolf, 1972). En diciembre de 1965, con el apoyo de la Universidad, rentó una casa como estación de campo en el pueblo de Tepetlaoxtoc, en el barrio de la Santísima, a donde fuimos a realizar una práctica de campo.

Antes de instalarnos en la casa, nos llevó durante varios sábados seguidos a conocer la región. Acabados los cursos, siguiendo el modelo de Kelly y de Vogt — director del Proyecto Chiapas de la Universidad de Harvard (Vogt, 1994)—, Ángel Palerm se trasladó a vivir a la casa de campo con nosotros los estudiantes durante dos meses ${ }^{16}$ en noviembre

\footnotetext{
${ }^{15}$ Agradezco a Cecilia O’Gorman sus comentarios y recuerdos sobre esta primera práctica.

16 Nos acompañaron durante semanas su esposa Carmen y sus hijos pequeños, Jacinta y Ángel. Integramos el grupo Cecilia O'Gorman, Margarita Campos, Jean Forbes, Sylvia Kurczyn, Teresa Robles, Beatriz Martínez Lavín, Rosalía Díaz Barriga, Cecilia Sánchez Barreda, Esther Izaguirre, Margarita Román Caballero, Alfonso Gortaire y yo.
} 


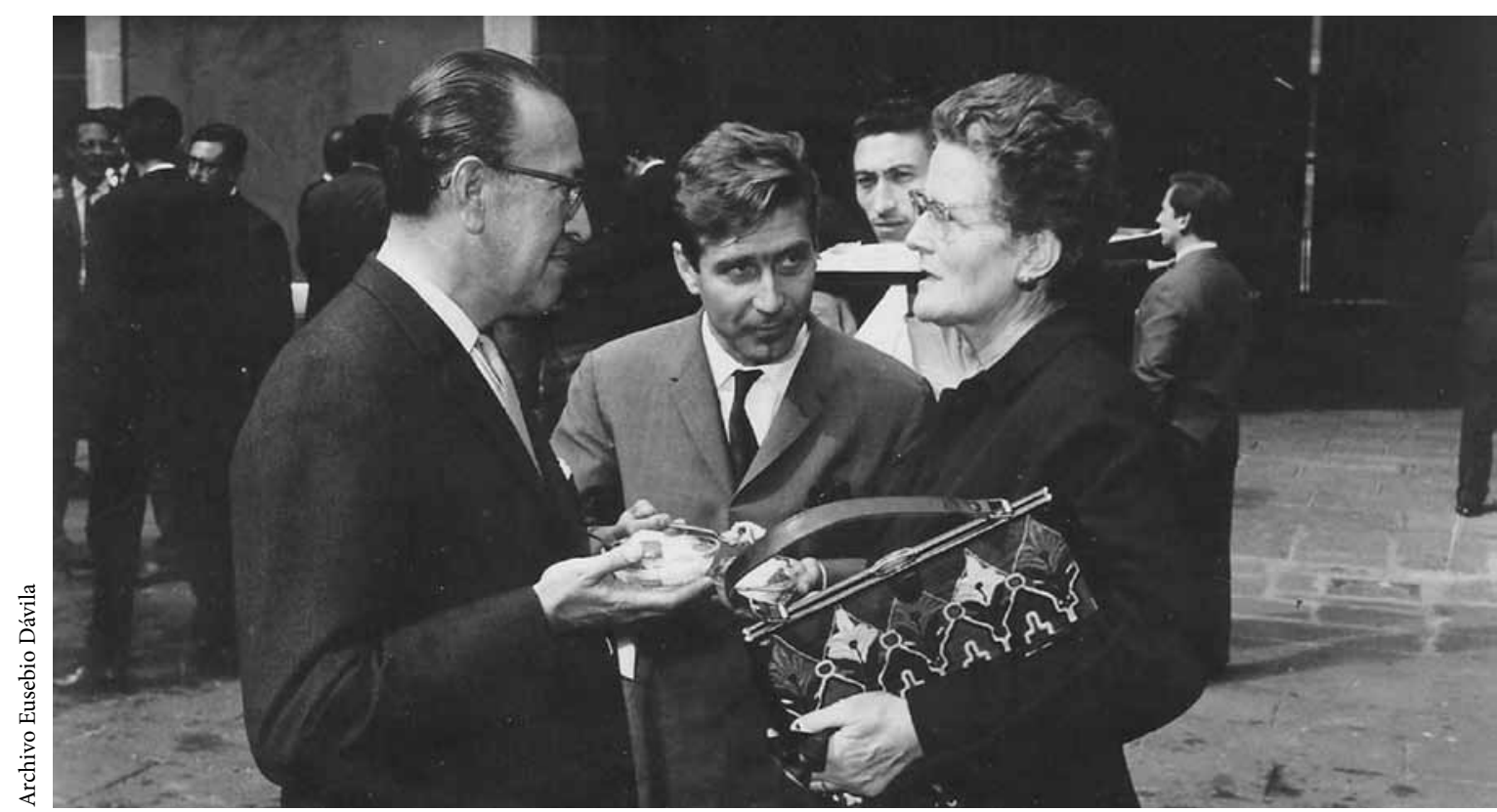

Isabel Kelly (en ese entonces representante de la Smithsonian Institution en México), Eusebio Dávalos Hurtado (en aquellas fechas director del INAH) y Santiago Genovés en la recepción por el XXV Aniversario del INAH. Patio del edificio del antiguo Museo de Antropología en la calle de Moneda, en la ciudad de México.

de 1965. A diferencia de los estudiantes de Kelly y de Vogt, nosotros pagábamos y preparábamos nuestros alimentos y no teníamos beca de la Universidad ni de otra institución. Otra gran diferencia con la dinámica del proyecto de Vogt, que Ángel Palerm mencionaba a menudo, era que mientras aquél repartía a los estudiantes en las comunidades, nosotros permanecimos todo el tiempo en la casa de campo, bajo la supervisión directa de nuestro profesor, como lo hacía Kelly.

Los primeros días de la práctica hicimos los recorridos de área. Antes revisamos los mapas y las fotos aéreas de la región para aprender a distinguir las zonas ecológicas que la integraban. Palerm consiguió este material en la Secretaría de la Defensa Nacional. Diario hacíamos un recorrido a una zona distinta: la llanura, el somontano y la sierra. De acuerdo con un decir suyo: "La antropología se hace con los pies", puesto que los recorridos implicaban "patear el terreno". Por ejemplo, la visita a San Jerónimo Amanalco, en la sierra, consistió en un trayecto de 20 kilómetros a pie. En el camino nos hacía ver la ecología, los sistemas agrícolas e hidráulicos, los asentamientos humanos y los restos arqueológicos. Constantemente nos preguntaba “¿qué viste?”. Era importante aprender la historia del lugar.

En las tardes teníamos que escribir en nuestro diario de campo y en fichas lo que habíamos visto. Lo hacíamos en máquina de escribir y dejábamos una copia en el archivo de la estación de campo. ${ }^{17}$ Clasificábamos las fichas con base en la Guía de Murdock, igual que lo hicieron los alumnos de Kelly. El objetivo del fichero común era construir un acervo de información sobre la región, disponible para todos aquellos que quisieran realizar investigación en ella. ${ }^{18}$

\footnotetext{
17 El archivo en papel se encuentra en la casa de campo de Tepetlaoxtoc. Calculamos que tiene alrededor de 35000 fichas acumuladas desde esa fecha. Desgraciadamente, en muy mal estado. Hasta 2012 se habían presentado en el posgrado de antropología de la UiA cuatro tesis doctorales y 28 de maestría que se han enriquecido con este material.

18 Vogt organizó un archivo con la información común del Proyecto Chiapas en la Universidad de Harvard (1994).
} 
Durante la tarde noche leíamos y discutíamos en un ambiente muy familiar lo observado a lo largo del día.

Después de haber realizado el recorrido de área, Palerm nos envió a establecer relaciones con la gente de Tepetlaoxtoc y los pueblos aledaños, así como a hacer genealogías y entrevistas con nuestros informantes. Otras tareas importantes fueron apreciar los edificios coloniales de los pueblos y acercarnos a sus archivos civiles y parroquiales para saber qué tipo de información estaba disponible, como él hizo con Kelly. La información recabada era sistematizada de la misma manera en que lo hacíamos con nuestras observaciones en los recorridos de campo. También debíamos describir los pueblos — sus edificios, servicios y comercios- e identificar los límites sociales y geográficos de los barrios y expresarlo en mapas. En la tarde escribíamos el diario y las fichas, para luego discutir y compartir la información obtenida. Durante estas sesiones el énfasis estaba en hacernos interrelacionar todos los factores.

Palerm, al igual que Kelly, revisaba nuestros diarios, genealogías, mapas y fichas y se sentaba con cada uno de nosotros para criticarlos y comentarlos. Era especialmente riguroso con la precisión y verificación de la información, así como con la redacción. Una anécdota sirve para documentar lo que para él era un dato preciso. En una ocasión reporté que "el maíz estaba chico”. Me preguntó “¿qué significa chico?". Como no contesté con la información precisa, a las 10:00 de la noche tuve que salir a medirlo y a averiguar si de acuerdo con la altura que mostraba, su desarrollo y el tiempo en que había sido plantado, su tamaño era el esperado o menor. En otras palabras, nos enseñó que en antropología no se emplean adjetivos calificativos y que los hechos y las observaciones deben fundamentarse con datos precisos.

Cuando consideró que conocíamos el área empezamos con las lecturas. Iniciamos con los artículos de Palerm y Wolf (1952, 1954,1955a, 1955b, 1957 y 1961) sobre la región. Era importante aprender historia regional a través de los cronistas y los códices Alva Ixtlixóchitl y el Tepetlaoxtoc. Al hacerlo, nos enseñó rudimentos de paleografía. Conforme nos adentrábamos en el campo, Palerm llevaba la literatura correspondiente a los diversos temas: parentesco, barrios, economía, demografía. Muchas veces nos obligaba a quedarnos en la casa a leer para poder discutir las lecturas durante las reuniones nocturnas como si fuera un seminario, como se había planteado en la reunión de Costa Rica que debía ser la enseñanza (Viqueira, 2000: 118).

A diferencia de Boas, interesado en el particularismo histórico, nos introdujo en el trabajo de la crítica y la creación teórica. La confrontación diaria entre lo observado y lo leído se orientaba a entender la región con base en la teoría de la evolución multilineal, muy cercana a los intereses de Kelly sobre la ecología y la historia. De acuerdo con nuestras observaciones, lecturas, inquietudes personales y la discusión colectiva, nos inducía a problematizar un tema que pudiera servir de base para realizar nuestra investigación personal. A diferencia del Proyecto Tajín, que en última instancia era el proyecto de la investigadora principal, Palerm nunca planteó nuestra investigación como suya, sino que quedó claro desde un principio que cada estudiante tenía su propio proyecto de investigación, de ser posible orientado a realizar su tesis de grado. A partir de que escogíamos un problema para investigar, nuestra pesquisa se enfocaba a buscar la información relacionada con el tema. Contábamos con los datos etnográficos generales del archivo para describir el entorno. La metodología de investigación era semejante, pero la discusión del tema mucho más rica (Viqueira, 2000: 121).

Por iniciativa suya, en 1966 la Universidad compró una casa en Tepetlaoxtoc, en el barrio de La Santísima, que se convirtió en la Casa de Campo "José de Acosta". ${ }^{19}$ Ese año se acondicionó para albergarnos y pudimos llevar a cabo una segunda práctica en invierno. Ésta fue distinta, más enfocada a investigar los problemas que habíamos elegido durante la práctica anterior. Del grupo inicial sólo

\footnotetext{
${ }^{19}$ La casa fue comprada con dinero de Palerm y la Universidad se la pagó después.
} 
tres optamos por realizar nuestras tesis de maestría en la región: Margarita Campos escogió estudiar la escuela en Tepetlaoxtoc, Cecilia O'Gorman se concentró en el sistema hidráulico y yo me decidí por la migración. Al final, Margarita y yo acabamos nuestras tesis dirigidas por Palerm. La de Margarita titulada "La escuela y la comunidad en un pueblo del Acolhuacan" y la mía "Asentamiento e historia demográfica: cuatro comunidades del Acolhuacan".20

El proceso de nuestras tesis implicó además un año de trabajo de campo en la zona, viviendo en la casa. Posteriormente escribimos los documentos correspondientes, que eran revisados por Palerm de manera constante y minuciosa, no sólo en cuanto a los planteamientos y la precisión de la información y de las fuentes, ya que también nos enseñó a estructurar el texto y a escribir correctamente el español. Con su apoyo nuestras tesis fueron publicadas, la de Margarita en la Colección SEP-Setentas (Campos, 1973) y la mía por la Secretaría de Educación Pública y el INAH (Pérez, 1975).

\section{REFLEXIONES FINALES}

En esta sección intentaré comparar este proyecto de enseñanza de la metodología de investigación de campo con sus antecesores, especialmente con Boas, Kroeber y Kelly. En primer lugar, destaco que la enseñanza del trabajo de campo en antropología implica un concepto de la propia disciplina. La información aquí vertida muestra que Palerm coincidía con sus precursores en conceptualizar la antropología como una disciplina de carácter empírico, en la que era fundamental la investigación de campo, la precisión de la información y su verificación. Las divergencias sobre qué datos obtener y su importancia conceptual surgen cuando se comparan los intereses y la orientación teórica de los investigadores.

${ }^{20}$ La UIA, a través de Palerm, nos dio la oportunidad de profundizar y ampliar nuestros estudios, así como de realizar tesis con nivel de maestría, de manera que el grado de maestro que otorgaba la Universidad estuviera realmente reflejado en nuestra formación (Palerm, 1988).
Sin embargo, los cuatro fundamentan sus planteamientos en la información empírica, es decir, en el trabajo de campo.

Sobra decir que los entornos históricos y geográficos de estos cuatro investigadores fueron distintos y que condicionaron su trabajo. Además aparecen diferencias en la metodología de investigación de campo, las relaciones institucionales, las relaciones con los estudiantes y los arreglos prácticos en el campo. En lo que se refiere a la metodología de la investigación en los cuatro destaca la permanencia durante largos periodos en las comunidades estudiadas con las que se establecían buenas y cercanas relaciones, en especial con los informantes clave, la importancia de la observación, las descripciones precisas del medio ambiente natural, las entrevistas, las genealogías, el levantamiento de censos y la sistematización de la información en diarios de campo. Es posible que la gran diferencia radique en lo referente a la sistematización de la información. Es Kelly, inspirada en Murdock, quien inicia la sistematización taxonómica en fichas de campo a partir de los diarios y notas. No obstante, en Kelly surge una modalidad de manejo de la información en grupo distinta a la experiencia de ir al campo en solitario, como lo hicieron Boas, Kroeber y sus discípulos. De acuerdo con sus cartas, además de recopilar y ordenar la información, ella supervisaba su precisión y su relevancia teórica en grupos de discusión con los estudiantes y colegas. Palerm reprodujo este modelo.

Al parecer, esta posibilidad está ligada a dos elementos: la personalidad del maestro y el contexto institucional. La literatura revisada revela que tanto Boas como Kroeber fueron personajes solitarios, exigentes, aunque alejados de sus estudiantes. Por otro lado, tanto Kelly como Palerm fueron también maestros exigentes, pero cercanos a sus alumnos y eso hizo posible formar generaciones de discípulos muy apegados a ellos y a su metodología. De acuerdo con la información proporcionada por Stocking, Boas pudo salir a campo financiado por una institución. Con base en esa experiencia, a lo largo de su vida promovió el desarrollo de la antropología en un marco institucional. Kroeber y Kelly recibieron 
financiamiento de instituciones y universidades estadounidenses. Al integrarse a la Smithsonian Institution con responsabilidades docentes, y a partir de su experiencia como estudiante, Kelly desarrolló un sistema para formar investigadores investigando. Bajo el amparo de la Smithsonian y de la ENAH organizó un seminario para revisar y discutir la bibliografía, la información de campo y los trabajos derivados. Los seminarios también fueron espacios donde se reclutaba y se formaba a los nuevos investigadores. Esto fue posible gracias al financiamiento de ambas instituciones, que se encargaban de pagar al maestro, la estación de campo y los gastos implicados, incluso los de los estudiantes. En el caso de Palerm, la UIA creyó en su proyecto e integró el trabajo de campo en el programa, lo contrató como maestro para ponerlo en práctica y compró una casa de campo a dónde llevar a los estudiantes. En el campo, enseñaba a recolectar información y supervisaba a los estudiantes, pero sobre todo discutía los datos en relación con la teoría, en grupo, como en seminarios. La diferencia con la experiencia de Kelly era que los estudiantes cubríamos nuestros gastos porque la Universidad no contaba con recursos para ello.

Muy ligada al trabajo institucional está la propiedad de los datos de campo. La información disponible demuestra que los estudiantes de Boas y Kroeber salían a campo a investigar problemas que interesaban a sus profesores, quienes empleaban los resultados en sus publicaciones, y además tenían el derecho de publicar personalmente los resultados de su trabajo, como lo hizo Kelly. En el caso de Tajín, el proyecto era de la institución patrocinadora, pero la información estaba disponible para todos los investigadores de la ENAH y del INAH que tuvieran interés en él. Si bien la investigación se publicó bajo la autoría de Kelly y Palerm, uno de los capítulos apareció firmado por el segundo. Respecto del Proyecto Chiapas de la Universidad de Harvard, la información del proyecto era propiedad de la Universidad, aunque estaba disponible con permiso de la institución y cada estudiante tenía la obligación de hacer un trabajo de investigación publicable de su autoría. En el caso del Proyecto del Acolhuacan de la UIA dirigido por Palerm, los datos debían permanecer en el archivo general, abierto a todos los estudiantes y profesores, pero los datos específicos eran propiedad de los estudiantes, quienes escribían y publicaban sus tesis. A diferencia del Proyecto Tajín, Palerm no publicó ningún trabajo sobre el mismo y era muy cuidadoso de respetar la propiedad intelectual de sus alumnos.

En lo que corresponde a las relaciones con los estudiantes, la información referente muestra a Boas y a Kroeber como maestros muy lejanos de los alumnos, en tanto que Kelly y Palerm aparecen como maestros muy cercanos a sus discípulos. La información disponible constata que la ENAH de los años cuarenta del siglo pasado y la UIA fueron espacios docentes en los que era posible que los maestros establecieran relaciones estrechas con los estudiantes. Esto implicaba reconocer y respetar las costumbres familiares prevalecientes en el medio social del que provenían. Así, Kelly reconoce haber fungido como chaperona de las estudiantes y Palerm tenía el cuidado de hablar con los padres de éstas para infundirles confianza sobre su trabajo y relaciones con ellas. Es más, para enfatizar el respeto que siempre hubo durante la práctica, invitaba a su esposa e hijos a convivir con los estudiantes.

Por último, incluyo algunas palabras sobre los arreglos cotidianos de las prácticas de campo de Kelly y Palerm. Ella se encargaba de seleccionar y contratar la casa de campo, comprar los víveres con dinero de la Smithsonian Institution y de llevar a su cocinero personal para la preparación de la comida y el mantenimiento de la casa. En el caso de la primera práctica con Palerm, él escogió y contrató la casa con apoyo de la Universidad, pero nos dejó la tarea del quehacer diario como una manera de hacernos responsables e involucrarnos en el trabajo común en el campo.

\section{BIBLIOGRAFÍA}

Alonso, Jorge, 2008, Ángel Palerm Vich, Colegio de Etnólogos y Antropólogos Sociales (Serie Biografías, núm. 1), México, en línea: <http://www.ceas.org.mx/ 
index.php?option $=$ com_content\&view $=$ article\&id $=228$ :angel-palem-vich\&catid $=36$ :destacados\&Itemid $=56>$.

Campos Calderón, Margarita, 1971, "La escuela y la comunidad en un pueblo del Acolhuacan", tesis de maestría, Universidad Iberoamericana, México.

, 1973, La escuela y la comunidad en Tepetlaoxtoc, Secretaría de Educación Pública (sEP-Setentas, núm. 89), México.

Fowler, Catherine S. y Robert V. Kemper, 2008, "Una vida en el campo: Isabel T. Kelly. 1906-1982”, en Isabel T. Kelly, Excavaciones en Chametla, Sinaloa, El Colegio de Sinaloa, Consejo Nacional para la Cultura y las Artes, México.

García Acosta, Virginia, 2000, "Presentación y semblanza de Ángel Palerm”, en Virginia García Acosta (coord.), La diversidad intelectual. Ángel Palerm in memoriam, Centro de Investigaciones y Estudios Superiores en Antropología Social, México, pp. 11-19.

Harris, Marvin, 1978, El desarrollo de la teoría antropológica. Una historia de las teorías de la cultura, Siglo XXI, México.

Kelly, Isabel T. y Ángel Palerm, 1952, The Tajin Totonac, The Smithsonian Institution, Washington, D. C.

Murdock, George Peter (dir.), 1963, Guía para la clasificación de datos culturales, Unión Panamericana, Washington.

Nutini, Hugo G., Pedro Carrasco Pizana y James M. Taggart, 1976, Essays on Mexican Kinship, The University of Pittsburgh Press, Pittsburgh.

Palerm, Ángel, 1967, Introducción a la teoría etnológica, Universidad Iberoamericana, México.

—_ 1987, Teoría etnológica, Universidad Autónoma de Querétaro, Querétaro.

_, 1988 , "Escuela de Antropología Social de la Universidad Iberoamericana", en Carlos García Mora (coord.), La antropología en México. Panorama histórico, vol. 7: Las instituciones, Instituto Nacional de Antropología e Historia (Colección Biblioteca del INAH), México, pp. 332-356.

- y Juan Vicente Palerm, 1976, "Introducción", en George Peter Murdock, Guía para la clasificación de los datos culturales, Universidad Autónoma de Querétaro, Querétaro, pp. 3-6.

— y Eric Wolf, 1952, "La civilización urbana", en Historia Mexicana, vol. II, núm. 2, El Colegio de México, pp. 184-209.

, 1954, "La distribución del regadío en el área central de Mesoamérica", en Ciencias Sociales, vol. V, núms. 25 y 26, pp. 2-15 y 64-74.

_ 1955a, "The Agricultural Basis of Urban Civilization in Mesoamerica", en Irrigation Civilizations: A Comparative Study. A Symposium on Method and Result in Cross-Cultural Regularities, Pan American Union (Social Science Monographs, núm. I), Washington, D. C., pp. 28-42. 1955b, "Irrigation in the Old Acolhua Domain, Mexico", en South Western Journal of Anthropology, Universidad de Nuevo México, vol. XI, núm. 3, pp. 265-281. _ 1957, "Ecological Potential and Cultural Development in Mesoamerica", en Studies in Human Ecology, Anthropological Society of Washington, Pan American Union (Social Science Monographs, núm. III), Washington, D. C., pp. 1-37.

__ 1961, "Sistemas de regadío prehispánico en Teotihuacán y en el Pedregal de San Ángel”, en Revista Interamericana de Ciencias Sociales, Segunda Época, vol. I, núm. 2.

— 1972, Agricultura y civilización en Mesoamérica, Secretaría de Educación Pública (sEP-Setentas), México.

Pérez Lizaur, María de la Soledad, 1970, "Asentamiento e historia demográfica: cuatro comunidades del Acolhuacan", tesis de maestría, Universidad Iberoamericana, México.

- 1975, Población y sociedad. Cuatro comunidades del Acolhuacan, Secretaría de Educación Pública, Instituto Nacional de Antropología e Historia, México.

Rutsch, Mechthild, 2007, Entre el campo y el gabinete. Nacionales y extranjeros en la profesionalización de la antropología mexicana (1877-1920), Instituto de Investigaciones Antropológicas-Instituto Nacional de Antropología e Historia, México.

Steward, Julian, 1966, The People of Puerto Rico, The University of Illinois Press, Urbana.

Stocking Jr., George W., 1983, Observers Observed. Essays on Ethnographic Fieldwork, The University of Wisconsin Press, Madison.

(ed.), 1996, Volkgeist as Method and Ethic. Essays in Boasian Ethnography and German Anthropological Tradition, The University of Wisconsin Press (History of Anthropology, vol. 8), Madison.

Viqueira Landa, Carmen, 2000, "Algunas aportaciones de Ángel Palerm a la enseñanza de la antropología”, en Virginia García Acosta (coord.), La diversidad intelectual. Ángel Palerm in memoriam, Centro de Investigaciones y Estudios Superiores en Antropología Social, México, pp. 115-126.

Vogt, Evon Z., 1994, Fieldwork among the Maya. Reflections on the Harvard Chiapas Project, University of New Mexico Press, Albuquerque.

\section{ARCHIVO}

The Smithsonian Institution, correspondencia entre Isabel T. Kelly y la Dirección del Instituto de Antropología Social, 1946-1952. 